\title{
Aerodynamic Interaction Studies on Amphibious Vehicle in Forward and Hovering Flight
}

\author{
P. Vikram, E. Balasubramanian*, M. Manova and G. Surendar \\ Centre for Autonomous System Research, \\ Vel Tech Rangarajan Dr Sagunthala R\&D Institute of Science and Technology, \\ Chennai, Tamilnadu, 600062, India \\ *Email: esak.bala@gmail.com
}

\begin{abstract}
Unmanned aerial vehicles (UAVs) have gained a lot of attention in recent times due to its versatility in deployment for multifaceted operations. The development of amphibious UAVs with inculcating the features of hovercraft and multi-rotor has tremendous impact on military, naval and coastal guard applications. Stability and performance of this kind of vehicle highly depend on aerodynamic interaction of multirotor with respect to various wind conditions. The present study focuses on performing computational fluid dynamic (CFD) analysis on examining the vortex formation, turbulent regimes, wake region, tip vortex formulation and ground effect. Preliminary flow analysis is performed to determine the angle of attack (AoA) and wind speed on which minimal drag is experienced by the amphibious structure. Further, analysis conducted through varying the relative velocity of the vehicle and changing the speed of the propellers. The pressure distribution across the fuselage and rotor surface predicted the stability of the vehicle. The ground effect is examined through varying the clearance between the vehicle and ground surface with respect to a multiplicity of rotor diameter. CFD analysis results suggested that at $5^{\circ} \mathrm{AoA}$ and $8.3 \mathrm{~m} / \mathrm{sec}$ the designed amphibious vehicle yielded superior performance characteristics and stability.
\end{abstract}

Keywords: Amphibious vehicle; multi-rotor interaction; co-efficient of thrust; advance ratio; induced inflow ration

\section{INTRODUCTION}

Unmanned aerial vehicles (UAVs) are predominantly utilised in diverse applications [1] including defence, surveillance, societal missions, environmental monitoring and disaster tasks. Compare to fixed and flapping wing UAVs, rotary-wing vehicles are having superior hovering characteristics which is very much needed in multifaceted environments. The aerodynamic behaviour of rotors is of great significance in controlling this kind of vehicles. The interaction between the rotor and the fluid domain is still a challenging problem. Performing computational fluid dynamic (CFD) analysis is considered to be interesting to study the vortex formation, wake region, tip vortex transition to turbulence regimes and multirotor interactions [2]. The effect of wind gust conditions is inherently complex due to the collision of tip vortices among the rotors and its own tip vortex. In addition, vortical wake flows, transonic flow regions, rotor blade dynamics and blade elastic deformation studies are performed [3]. The velocity difference between advancing and retreating side of rotors may create unbalance of the vehicle with reference to pitching or rolling moment [4]. The effect of wake on rotor flow field has significant effect on the performance of the vehicle. Induced velocity and pressure regimes due 
to the wake with intense vorticity of flow affects the lift characteristics [5] and determining the flow field is of greater significance. It is necessary to perform CFD analysis to investigate the rotor wake regions, tip vortices and turbulence phenomenon.

In this work, a novel amphibious vehicle is considered to examine the aerodynamic interactions. The vehicle is designed through inculcating the features of multirotor and hovercraft. A water sample mechanism is placed in the middle region of the vehicle. The complex geometry of the vehicle causes intricate flow fields, vorticity and turbulence regimes and solving multiphysics problem is also crucial. Investigations on helicopter rotor unsteady aerodynamics are well known topic of research. Antoniadis et al. [6] performed CFD analysis for various flight conditions (pitch up, cruise, dynamic stall and high-speed tail shake) and computed surface pressure co-efficient. Experimental results are compared with CFD analysis and they are in good agreement in most cases. Biava et al. [7] compared the numerical analysis with experimental results of unsteady sensor positions of full helicopter model at various crosssectional planes of the fuselage. They have evaluated the pressure on rotors and fuselage and their fluid-structure conditions are examined. Boyd et al. [8] conducted numerical investigations of unsteady aerodynamics of helicopter rotor blade through combining rotor loading and rotor flow field models. Cao et al. [9] investigated the wake effect of a rotor in hovering mode and semi-empirical correction for the vortex core effect is simulated. Conlisk [10] studied the wake characteristics of helicopter rotor blade in hovering condition. The blade vortex interactions and aeroelasticity of blades are evaluated experimentally. Dindar et al. [11] incorporated adaptive finite element method to study the regions affected through vortical flows of rotor blades. A topology-based vortex core detection technique in combining with interpolation error estimate is employed to determine the vortex flow characteristics of rotor blades. Domenge and llie [2] performed numerical investigations to examine the blade-vortex interaction (BVI) phenomenon of rotorcraft using potential flow theory. Felismina et al. [12] evaluated the aerodynamic performance of UAV with seeder assembly for various climbing angle and corresponding drag force is measured using CFD analysis. Filippone and Michelsen [13] carried out the drag estimation of helicopter fuselage for various configurations and corresponding centre of pressure regimes are determined. Flow visualization studies are conducted using wind tunnel for asymmetric flow conditions and varied geometrical conditions. Qijun et al. [5] predicted the unsteady aerodynamic flow characteristics of helicopter rotors using spatial and temporal discretization methods.

Various mathematical schemes such as Roe-WENO, JST and Roe-MUSCL are employed to determine convective fluxes. Shi et al. [14] developed a viscous wake model (VWM) to predict the rotor wake dynamics and structural deformation of the helicopter blade. In addition, chordwise pressure distribution and span wise measurement of force of rotor blade is performed. Steijl and Barakos [15] conducted aerodynamic interaction studies for the case of fuselage, tail rotor and main rotor of a helicopter. The effect of rotor loading and estimation of pressure co-efficient at varied azimuth angle is determined. Tan and Wang [3] studied the unsteady aerodynamic rotor wake of helicopter blade using full span free wake method combination with unsteady panel and the CFD analysis results are validated with experimentation. However, very few studies are performed for analysing the aerodynamic performance characteristics of quadcopter. Lopez et al. [16] studied the flow around a quadcopter propeller using SA and K- $\omega$ models. The wake regions, velocity and pressure fields and performance coefficients of propeller are determined for different rotational velocities using moving reference frame (MRF) method. Thibault et al. [17] captured vortex phenomenon of quadcopter using Lattice-Boltzmann and LES turbulence modelling methodologies. Yoon et al. [18] analysed the effect of separation among the four rotors, fuselage and wings on hovering 
condition. Smaller the separation distance between the rotors causes decrease inefficiency. In contrast to existing studies, present work focuses on examining the multirotor interactions, rotor-fuselage wake regions of a novel amphibious vehicle. A multi-rotor UAV with hovercraft configuration that can land and glide on water surface to collecting water samples is defined as amphibious UAV. For water quality evaluation of large and inaccessible water bodies, amphibious vehicle provides effective and rapid solutions where the intervention of human is difficult and also to avoid using boats. Water sampler mechanism shown in Figure1 is used to collect the water samples and subsequently stored in a container. In addition water quality sensors such as $\mathrm{pH}$, turbidity, dissolved oxygen and electrical conductivity are assembled into a case as a unit which will be used to perform in-situ water quality measurement. The amphibious vehicle is aimed to hover and cruise for the desired missions and it suffers for low aerodynamic efficiencies. Accurate prediction of flow characteristics around the amphibious vehicle is paramount of interest and considered to be challenging for CFD analysis. The mission of amphibious UAV has various phases such as vertical takeoff, landing on the water and takeoff from the surface of water. However, the present work focuses on vertical take-off and forwarding flight rotors interaction. CFD studies are performed through varying the relative velocity of vehicle and speed of the propellers. In addition, ground effect is examined and wake regions are determined.

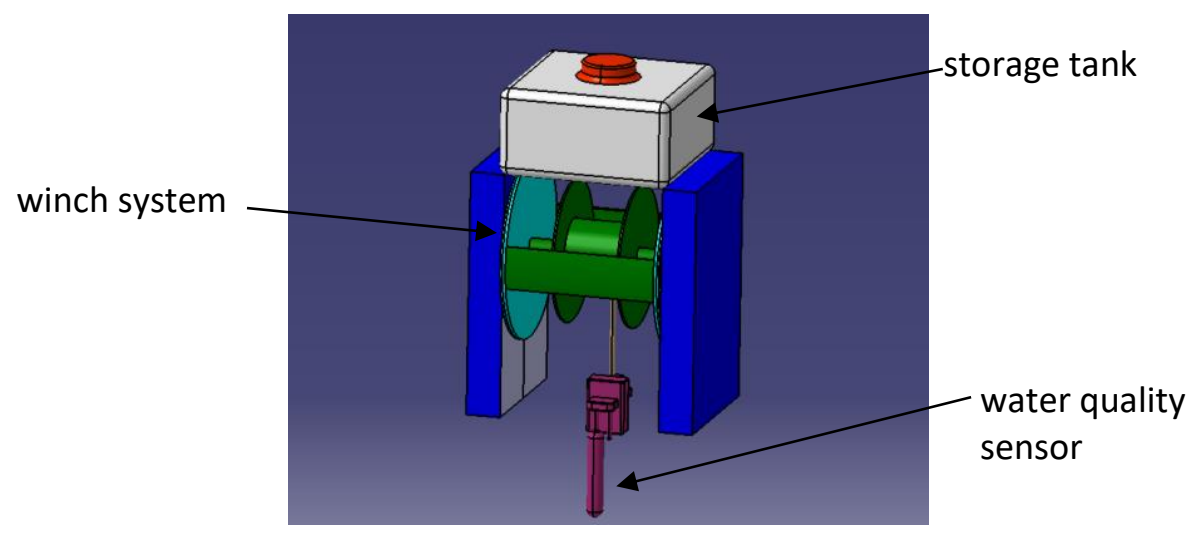

Figure 1. Water sampler mechanism.

\section{COMPUTATIONAL DOMAIN}

The conceptual model of amphibious vehicle is designed in CATIA V5 platform and simulations are performed using ANSYS FLUENT V16.2. Geometrical size of the quadcopter is $1.2 \times 0.6 \mathrm{~m}$, length of the arm $1.04 \mathrm{~m}$ and the total weight is $28.88 \mathrm{~kg}$. In order to eliminate the wake blockage and account for wall effect and buoyancy effect in CFD analysis, the computational domain shown in Figure 2 is 10 times larger than the amphibious UAV model. Buoyancy effect is one of the tunnel effects occur in the computational domain. It depends upon the size of domain. If it is a small domain, boundary layer thickness will increase. Due to which, variation in static pressure along the axis of the model takes place. It will cause an increase in drag which is known as buoyancy effect.

Due to the incompressible fluid analysis, the pressure-based solver is used to perform necessary calculations, with second-order upwind discretization for convective and diffusive terms. All the simulations are performed in the atmospheric conditions having air density of 1.2 $\mathrm{kg} / \mathrm{m}^{3}$ and atmospheric pressure. Furthermore, dynamic viscosity of $1.85 \times 10^{-5} \mathrm{~kg} / \mathrm{m} . \mathrm{s}$ and a temperature of $30^{\circ} \mathrm{C}$ are taken into consideration. For each condition, convergence criteria are 
increased up to 1 xe-05 to increase the accuracy of solution. Each simulation took 5000 iterations to stabilize all the governing equations and also attaining convergence of the solution. The MRF model is created in Figure 3 to vary the speed of multirotor. A domain is introduced to simulate the rotor flow characteristics around the propeller is known as moving reference frame (MRF) as shown in Figure 3.
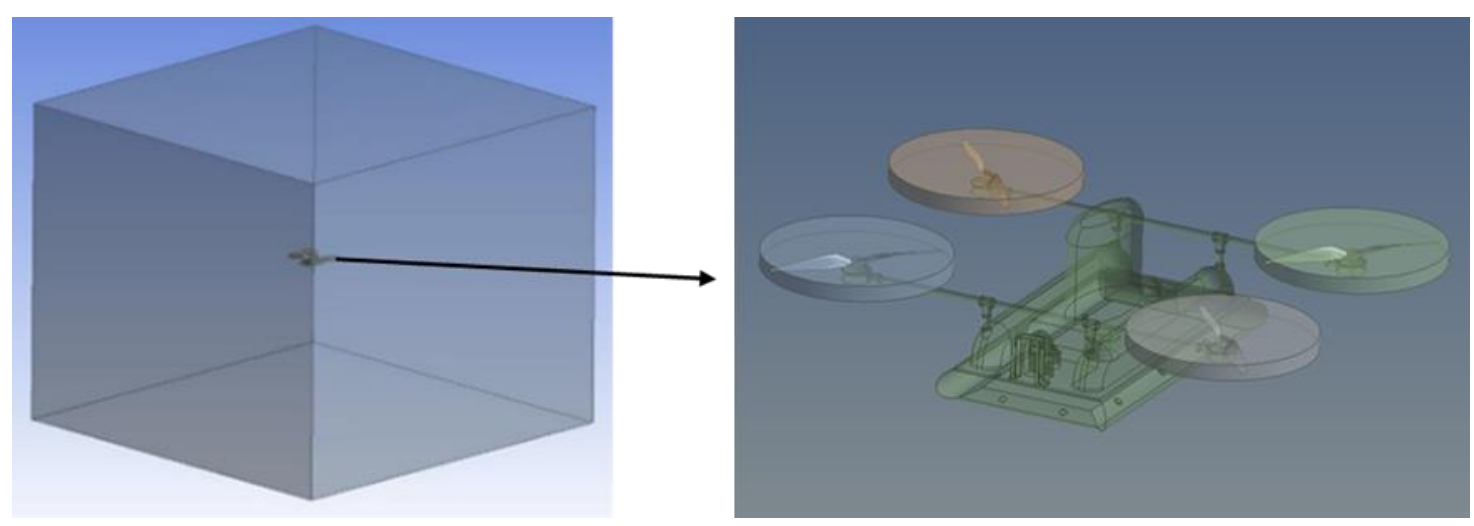

Figure 2. Computational domain.

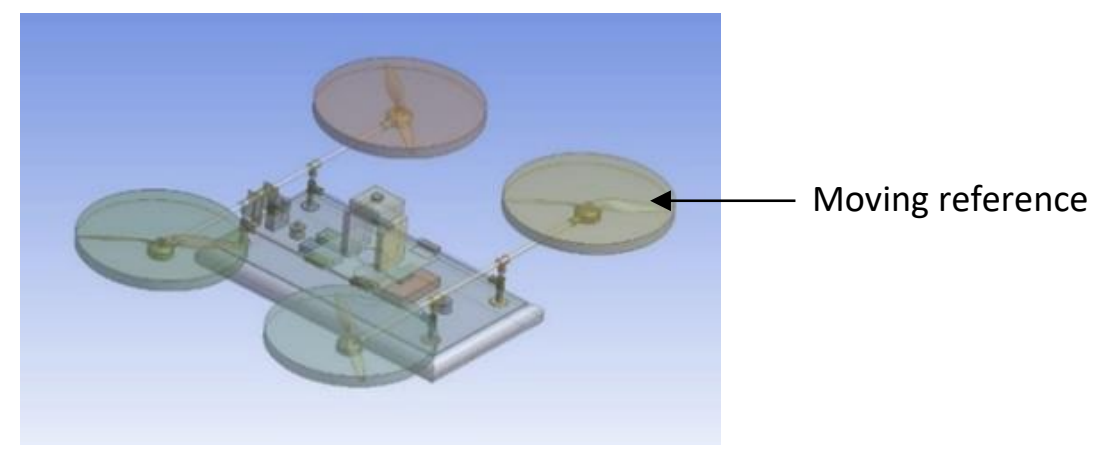

Figure 3. UAV with MRF domain.

\section{Mesh Generation}

The tetrahedral mesh is created for the entire fluid-structure domain using ANSYS ICEM tool for calculating high Reynolds number flow regimes. The total number of elements in the computational grid is approximately 17, 00,000 and the mesh quality is evaluated through performing orthogonality and skewness check (Figure 4). It is also observed from Figure 5 that most of the elements are lying in the region of $0.85-0.90$ in orthogonality and skewness values of $0.05-0.35$ (Figure 6) and hence mesh quality is quite satisfactory to examine the intricate profiles of amphibious structure. Grid independence test is conducted to ensure the convergence of solution for greater accuracy.

\section{Numerical Approach or Modelling}

The turbulent flows are usually computed through solving Reynolds-averaged Navier-Stokes equations (RANS). The generation numerical modelling consists of defining the appropriate geometry, grid generation and solve for necessary boundary conditions [19]. Since the amphibious structure is complex and capturing the turbulent flow characteristics is crucial for 
the success of selecting CFD model. Using RANS with finite mesh generation and small-time steps turbulent phenomenon can be captured and it minimizes computation burden through solving the statistically averaged equation system. In this study three turbulent models such as $\mathrm{k}-\varepsilon, \mathrm{k}-\varepsilon$ and shear stress transport (SST) $\mathrm{k}-\omega$ are initially evaluated for its performance. $\mathrm{k}-\varepsilon$ models is a two transport equations model in which turbulent dissipation $\varepsilon$ evaluate the turbulent characteristics [20]. This model accounts buoyancy, viscous heating and compressibility and it may not be suitable for large pressure gradients. A k- $\omega$ model [20] where turbulence kinetic energy $k$ and the specific dissipation rate $\omega$ is incorporated to predict the turbulent flow characteristics of amphibious UAV. This model envisages obtaining wake regions, boundary layers around the amphibious UAV, reverse flow regions, turbulent kinetic energy and dissipation rate. In addition, shear stress transport (SST) $\mathrm{k}-\omega$ model [21] which is similar to the standard k- $\omega$ model is utilised to calculate the near-wall flow characteristics using a blending function. It also estimates the flow properties away from the wall surface. In the SST k- $\omega$ model, a damped cross-diffusion term is used to determine the turbulent shear stress through modifying the turbulent viscosity which ensures the selected model is more accurate and reliable for diverse flow fields.

In the literature, $k-\varepsilon, k-\omega$ and shear stress transport (SST) $k-\omega$ turbulent models are widely used to examine the flow characteristics of UAV [16]. In order to predict the near-wall flow characteristics, Celic and Hirschel [21] considered SST k-omega model and the results were promising for diverse flow fields. However, they have not conducted comparative evaluation to determine the lift and drag co-efficient of three turbulent models. Hence, the present study is made to compare them. Since k- $\omega$ model is a superset of SST k- $\omega$ model that is considered as reference model in this study. In addition, comparative evaluation of $\mathrm{k}-\varepsilon, \mathrm{k}-\omega$ and shear stress transport (SST) k- $\omega$ models provided greater understanding the turbulent characteristics of amphibious UAV in predicting the drag force. The following are the general equations used to solve the turbulent models [19].

\section{Transport equation for the standard $k$-E model}

For turbulent kinetic energy $(\mathrm{k})$

$$
\frac{\partial}{\partial t}(\rho k)+\frac{\partial}{\partial x_{i}}\left(\rho k u_{i}\right)=\frac{\partial}{\partial x_{j}}\left[\left(\mu+\frac{\mu_{t}}{\sigma_{k}}\right) \frac{\partial k}{\partial x_{j}}\right]+P_{k}+P_{b}-\rho \varepsilon-Y_{M}+S_{k}
$$

For dissipation $(\varepsilon)$

$$
\frac{\partial}{\partial t}(\rho \varepsilon)+\frac{\partial}{\partial x_{i}}\left(\rho \varepsilon u_{i}\right)=\frac{\partial}{\partial x_{j}}\left[\left(\mu+\frac{\mu_{t}}{\sigma_{\varepsilon}}\right) \frac{\partial \varepsilon}{\partial x_{j}}\right]+C_{1_{\varepsilon}} \frac{\varepsilon}{k}\left(P_{k}+C_{3_{\varepsilon}} P_{b}\right)-C_{2_{\varepsilon}} \rho \frac{\varepsilon^{2}}{k}+S_{\varepsilon}
$$

Transport equation for the standard $k-\omega$ model

Kinematic eddy viscosity

$$
v_{T}=\frac{k}{\omega}
$$

Turbulent kinetic energy

$$
\frac{\partial k}{\partial t}+U_{j} \frac{\partial k}{\partial x_{i}}=T_{i j} \frac{\partial U_{i}}{\partial x_{j}}-\beta^{*} k \omega+\frac{\partial}{\partial x_{j}}\left[\left(v+\sigma^{*} v_{T}\right) \frac{\partial k}{\partial x_{j}}\right]
$$


Specific dissipation rate

$$
\frac{\partial \omega}{\partial t}+U_{i} \frac{\partial \omega}{\partial x_{i}}=\alpha \frac{\omega}{k} T_{i j} \frac{\partial U_{i}}{\partial x_{j}}-\beta \omega^{2}+\frac{\partial}{\partial x_{j}}\left[\left(v+\sigma v_{T}\right) \frac{\partial \omega}{\partial x_{j}}\right]
$$

Shear stress transport (sst) $k$ - $\omega$ model

Kinematic eddy viscosity

$$
v_{T}=\frac{\alpha_{1} k}{\max \left(\alpha_{1} k, S F_{2}\right)}
$$

Turbulent kinetic energy

$$
\frac{\partial k}{\partial t}+U_{j} \frac{\partial k}{\partial x_{j}}=P_{k^{-}} \beta^{*} k \omega+\frac{\partial}{\partial x_{j}}\left[\left(v+\sigma_{k} v_{T}\right) \frac{\partial k}{\partial x_{j}}\right]
$$

Specific dissipation rate

$$
\frac{\partial \omega}{\partial t}+U_{i} \frac{\partial \omega}{\partial x_{i}}=\alpha S^{2}-\beta \omega^{2}+\frac{\partial}{\partial x_{j}}\left[\left(v+\sigma_{\omega} v_{T}\right) \frac{\partial \omega}{\partial x_{j}}\right]+2\left(-F_{1}\right) \sigma_{\omega^{2}} \frac{1}{\omega} \frac{\partial k}{\partial x_{i}} \frac{\partial \omega}{\partial x_{i}}
$$

The present study considers frozen rotor approach by Lopez et al. [16] to simulate the rotor flow characteristics. An interface zone is established between static frame and MRF which is used to calculate fluxes at the boundary of the adjacent zone. However, the rotor geometry is fixed for the computation which is analogous to freezing the motion of the moving part in a specific position and observing the instantaneous flow field with the rotor in that position. The conservative equations for an MRF assuming incompressible and steady flow equations are,

$$
\begin{aligned}
& \nabla \cdot \overrightarrow{v_{r}}=0 \\
& \rho \nabla \cdot\left(\overrightarrow{v_{r}} \vec{v}+\rho(\vec{\omega} \times \vec{v})=-\nabla p+\nabla \overline{\bar{\tau}}+\vec{F}\right.
\end{aligned}
$$




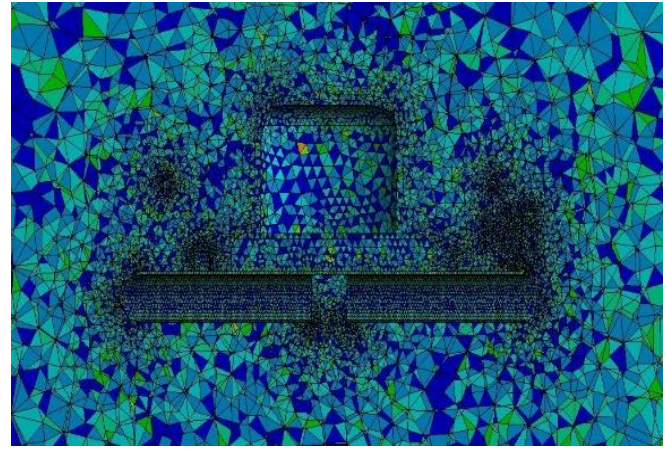

(a) Model with canopy skewness

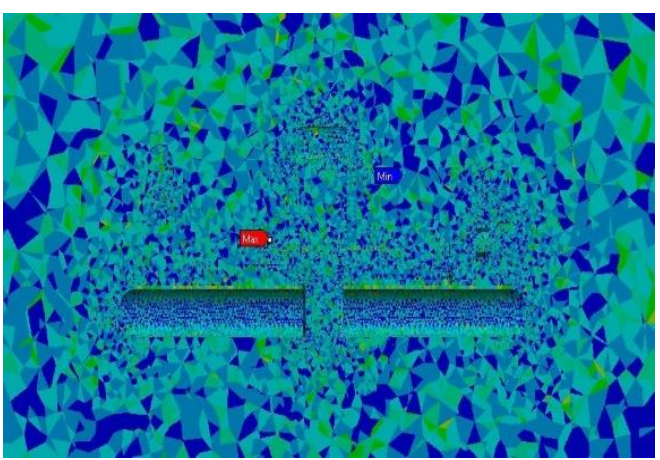

(c) Model without canopy skewness

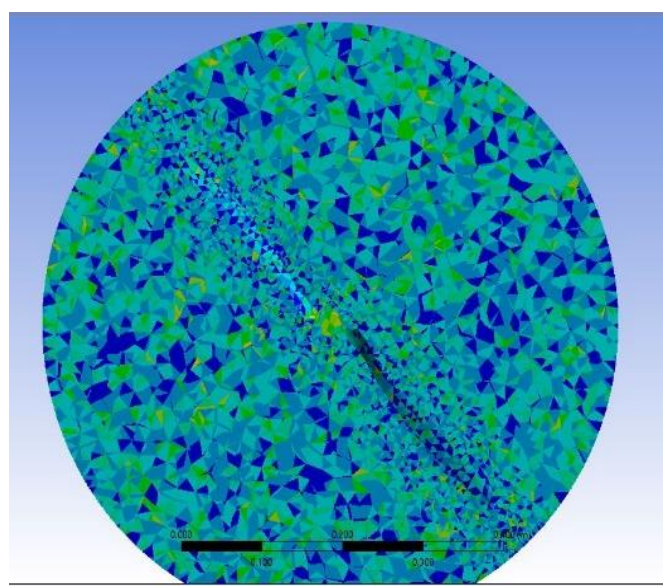

(e) Skewness check of MRF domain

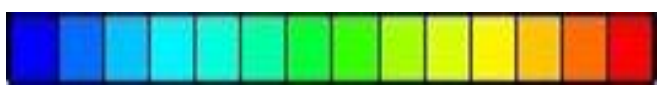

0

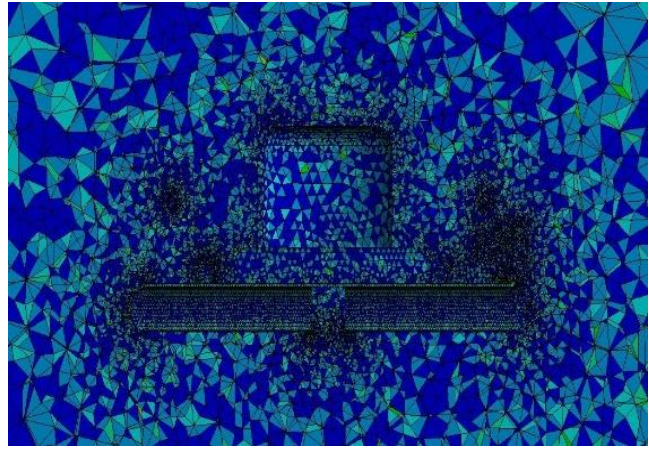

(b) Model with canopy orthogonality

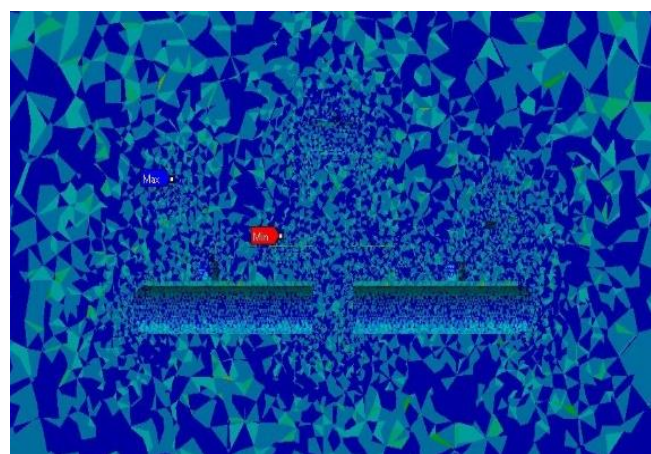

(d) Model without canopy orthogonality

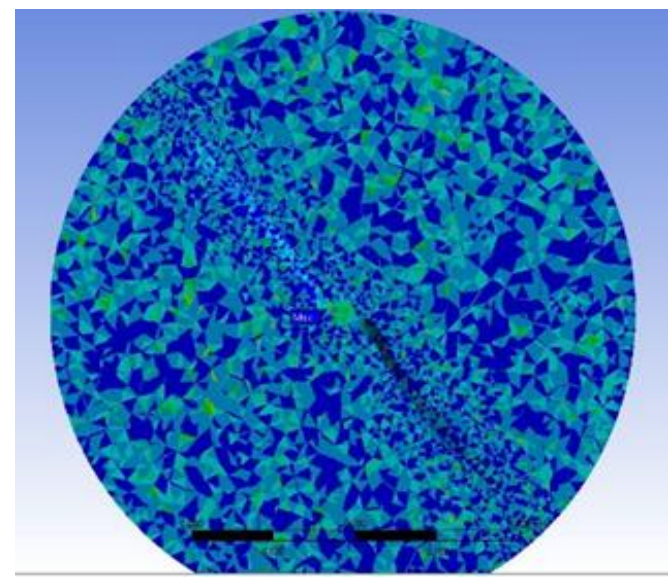

(f) Orthogonality check of MRF domain

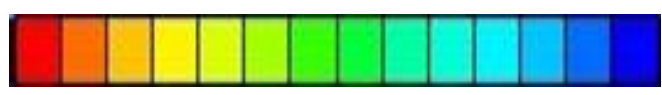

1

Figure. 4. Skewness and orthogonality check.

\section{NUMERICAL ANALYSIS WITHOUT AND WITH CANOPY}

The aerodynamic performance of the amphibious vehicle is significantly influenced by unsteady flow characteristics around the vehicle due to the complex integration of rotor and fuselage. The vehicle has two important manoeuvring phases i.e. hovering and forwards flight phase. In this vehicle, hovering is essential to collect the water samples from the remote water resource and forward flight conditions to move towards desired location. 

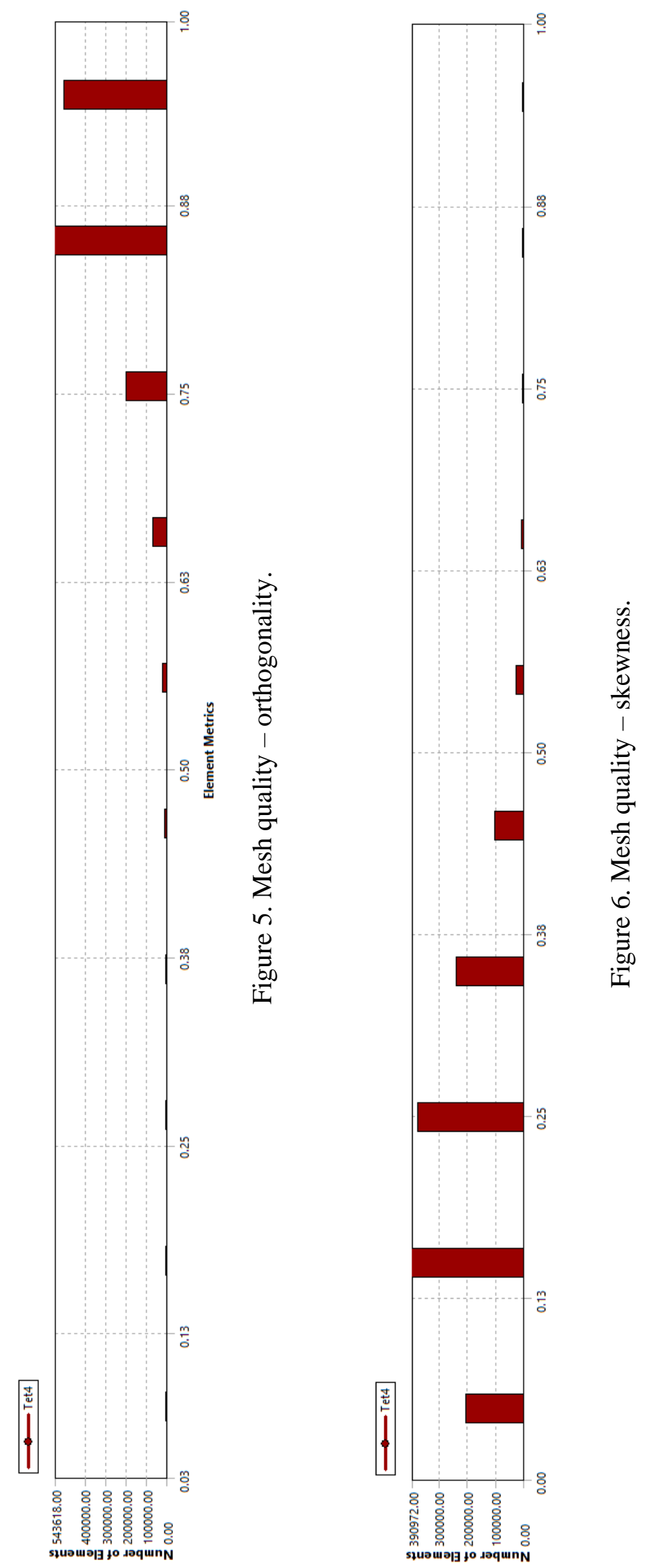
The preferred operating condition of amphibious vehicle is at $30 \mathrm{~km} / \mathrm{hr}(8.3 \mathrm{~m} / \mathrm{sec})$ during manoeuvring. However, evaluating the aerodynamic characteristics above and below $8.3 \mathrm{~m} / \mathrm{sec}$ may provide a significant estimation of thrust. Hence we have considered $5 \mathrm{~m} / \mathrm{sec}$ and $10 \mathrm{~m} / \mathrm{sec}$ as lower and upper limit of vehicle speed and corresponding CFD analysis is performed. Hence, these conditions are simulated for with and without canopy configurations. Each simulation in the analysis is taken 16-20 hours to converge. To improve the accuracy of the solution, convergence criteria is increased up to $10 \mathrm{e}-05$ of each governing equation. Preliminary CFD analysis through varying the angle of attack (AoA) and wind speed conditions are performed. It is evident from Table 1 that at $5^{\circ}$ of AoA and $8.3 \mathrm{~m} / \mathrm{sec}$ wind velocity, maximum co-efficient of lift is obtained and hence this condition is considered for further analysis. In addition, simulations are performed to estimate the drag for various turbulent models and the results suggested that (Table 2) SST k- $\omega$ model obtained minimal error and hence it is accounted in numerical studies for examining the aerodynamic interaction studies.

Table 1. Amphibious UAV in isolated fuselage condition.

\begin{tabular}{lccccc}
\hline \multirow{2}{*}{ Wind speed $(\mathrm{m} / \mathrm{s})$} & \multirow{2}{*}{ Parameters } & \multicolumn{4}{c}{ AoA } \\
\cline { 3 - 6 } & & $0^{\circ}$ & $5^{\circ}$ & $8^{\circ}$ & $10^{\circ}$ \\
\hline 5.0 & $\mathrm{C}_{\mathrm{D}}$ & 0.489 & 0.346 & 0.364 & 0.382 \\
& $\mathrm{C}_{\mathrm{L}}$ & 0.165 & 0.172 & 0.269 & 0.285 \\
& Drag & 1.320 & 1.270 & 1.327 & 1.529 \\
\hline 8.3 & $\mathrm{C}_{\mathrm{D}}$ & 0.489 & 0.352 & 0.364 & 0.382 \\
& $\mathrm{C}_{\mathrm{L}}$ & 0.165 & 0.176 & 0.272 & 0.289 \\
& Drag & 3.639 & 3.582 & 3.654 & 4.213 \\
\hline 10.0 & $\mathrm{C}_{\mathrm{D}}$ & 0.489 & 0.349 & 0.367 & 0.379 \\
& $\mathrm{C}_{\mathrm{L}}$ & 0.201 & 0.222 & 0.280 & 0.289 \\
& Drag & 5.280 & 5.157 & 5.358 & 6.059 \\
\hline
\end{tabular}

Table 2. Comparison of various turbulent models in predicting drag characteristics and determination of error.

\begin{tabular}{lcccccc}
\hline $\begin{array}{l}\text { AoA } \\
\left({ }^{\circ}\right)\end{array}$ & $\begin{array}{c}\text { Relative } \\
\text { velocity } \\
(\mathrm{m} / \mathrm{s})\end{array}$ & $\begin{array}{c}\text { Drag }(\mathrm{N}) \\
(\mathrm{k}-\omega \\
\text { model })\end{array}$ & $\begin{array}{c}\text { Drag (N) } \\
(\mathrm{k}-\varepsilon \text { model })\end{array}$ & $\begin{array}{c}\text { Drag }(\mathrm{N}) \\
(\mathrm{SST} k-\omega \\
\text { model })\end{array}$ & $\begin{array}{c}\text { Error } \\
(\mathrm{k}-\omega / \mathrm{SST} \\
\mathrm{k}-\omega)\end{array}$ & $\begin{array}{c}\text { Error } \\
(\mathrm{k}-\omega / \mathrm{k}-\varepsilon)\end{array}$ \\
\hline \multirow{2}{*}{5} & 5 & 3.615 & 3.639 & 3.560 & -0.055 & -0.024 \\
& 8.3 & 3.582 & 3.577 & 3.593 & 0.0011 & 0.005 \\
\hline \multirow{2}{*}{8} & 5 & 3.654 & 3.743 & 3.701 & 0.047 & -0.089 \\
\hline
\end{tabular}

Table 3. Power calculation for the various flight condition shown.

\begin{tabular}{lcccc}
\hline \multirow{2}{*}{ Wind Speed $(\mathrm{m} / \mathrm{s})$} & \multicolumn{4}{c}{ Power required $(\mathrm{W})$} \\
\cline { 2 - 5 } & $0^{\circ}$ & $5^{\circ}$ & $8^{\circ}$ & $10^{\circ}$ \\
\hline 5 & 1778.18 & 1206.96 & 1806.21 & 2419.20 \\
8.3 & 2951.78 & 1992.00 & 2732.88 & 3334.98 \\
10 & 2919.40 & 1975.46 & 2872.86 & 3986.02 \\
\hline
\end{tabular}


The present analysis considered negative $A o A$ and $C_{L}$ value decrease for airfoil sections. Since the multirotor system is a blunt body, $\mathrm{C}_{\mathrm{L}}$ value increases. A similar phenomenon is on aerodynamic analysis of helicopter fuselage was observed by Filippone [13]. The power requirement for varied wind speed conditions and AoA is given in Table 3. Due to the increase in AoA of above $5^{\circ}$, the predominant drag effect increases power requirement.

\section{CFD Analysis without Canopy}

\section{Hovering Phase}

In the hovering phenomenon, all rotors generate an equal amount of thrust for the given constant speed of rotors rotating at $5000 \mathrm{rpm}$ using MRF model. Inlet wind velocity of $0.5 \mathrm{~m} / \mathrm{sec}$, turbulent viscosity ratio is maintained as 10 and atmospheric pressure condition is simulated. Simulation results predicted the airflow interference between rotors and fuselage and they are at minimal level. Thrust force generation is uniform from each rotor as seen in Figure 7(a) which ensures the hovering capability of the vehicle. The airflow around the propeller is disturbed due to the interference of main body structure. There is more pressure generation at the blade near to main body and it is reduced further apart from the fuselage as observed in Figure 7(b). While hovering, upstream flow got stagnated on the surface of the UAV and it induces the stagnation pressure rise due to adverse pressure gradient which is shown in Figure $7(\mathrm{c})$.

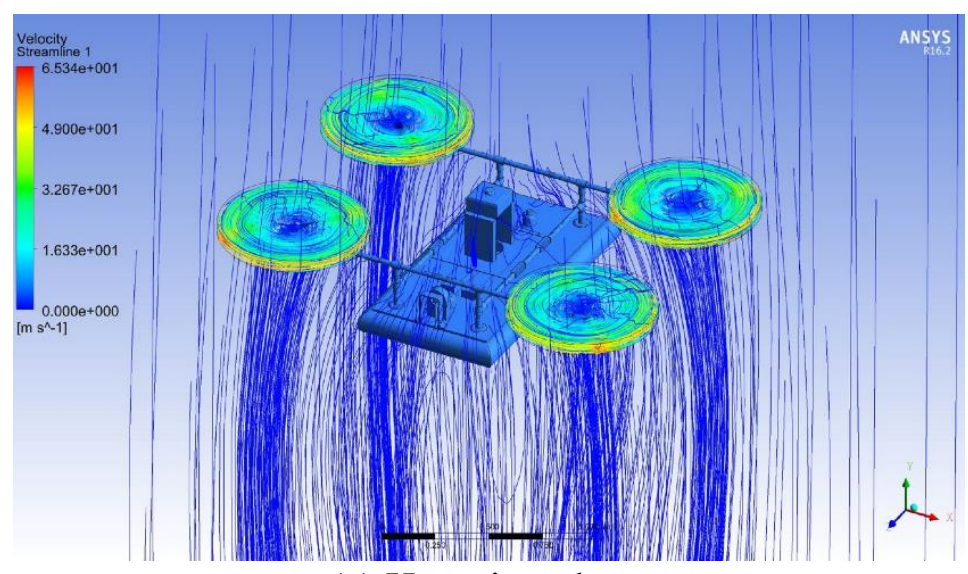

(a) Hovering phase

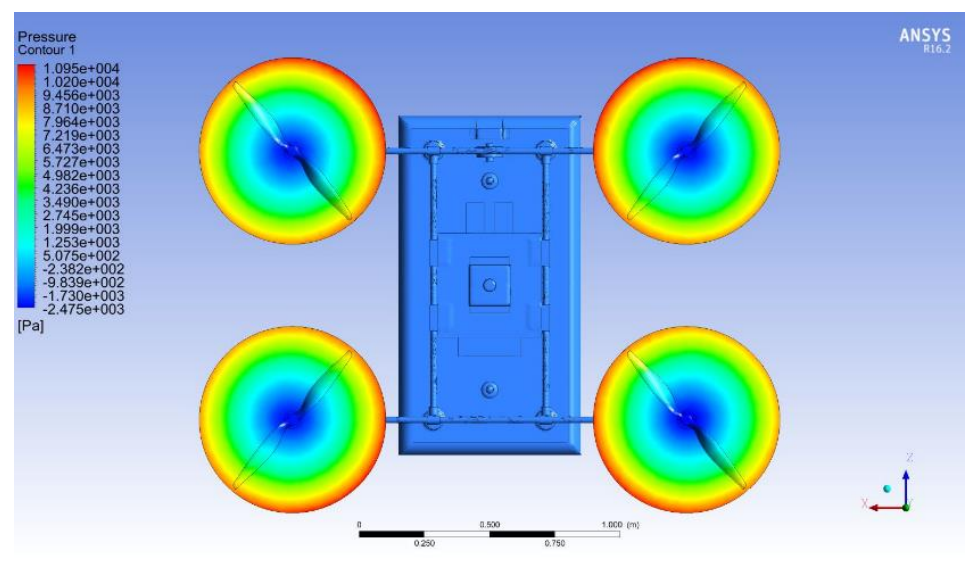

(b) Pressure contour 


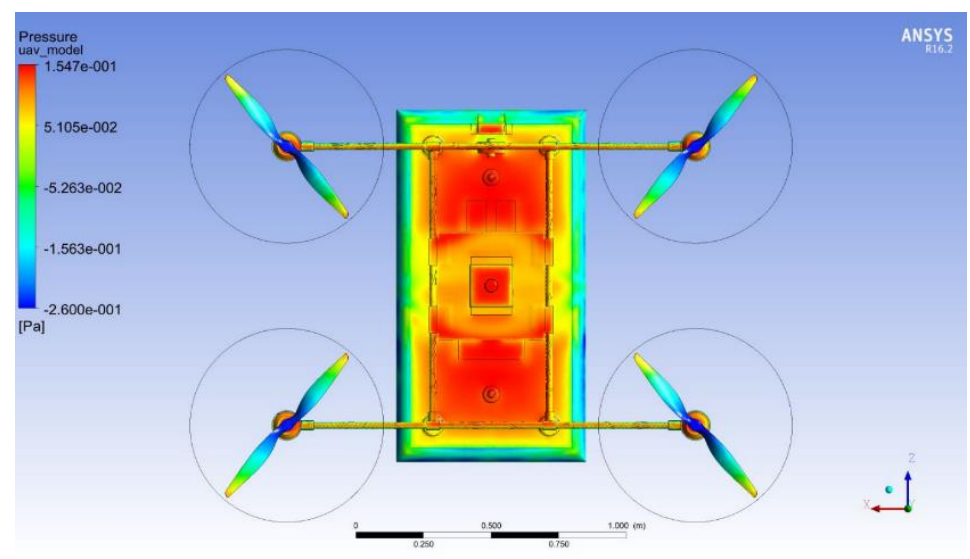

(c) Pressure contour on UAV surface

Figure 7. Amphibious UAV in hovering flight.

\section{Forward Flight}

The amphibious vehicle model is kept in the computational domain with a relative velocity of $8.3 \mathrm{~m} / \mathrm{sec}$ at $5^{\circ} \mathrm{AoA}$ and atmospheric pressure condition is considered for achieving the forward flight. It is evident from Figure 8(a) that, at the tip of propeller maximum velocity is observed than at the root. The rotation of rotor velocity is uniformly distributed as seen in Figure 8a. Also, due to the interference of main body structure with propellers, a region near to the body is obtained more pressure and it is reduced further apart as observed in Figure 8(b). There seems to be more flow interference between rotors and fuselage as observed in Figure 8(c). Also, high reverse flow region is created in the downstream of flow which can be clearly seen in Figure 8(c) which may affect the stability of UAV and similar phenomenon is also observed in hovering condition in Figure7(c).

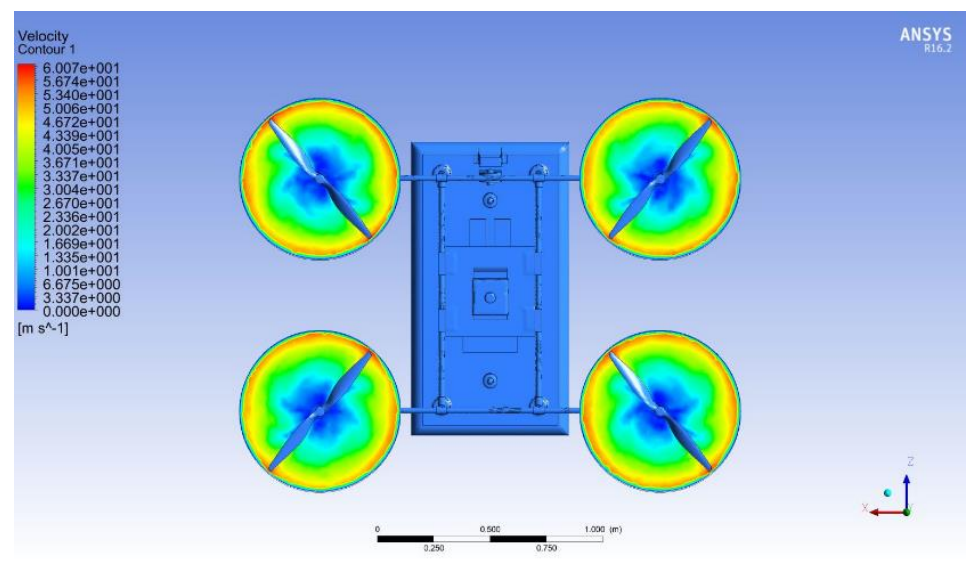

(a) Velocity contour 


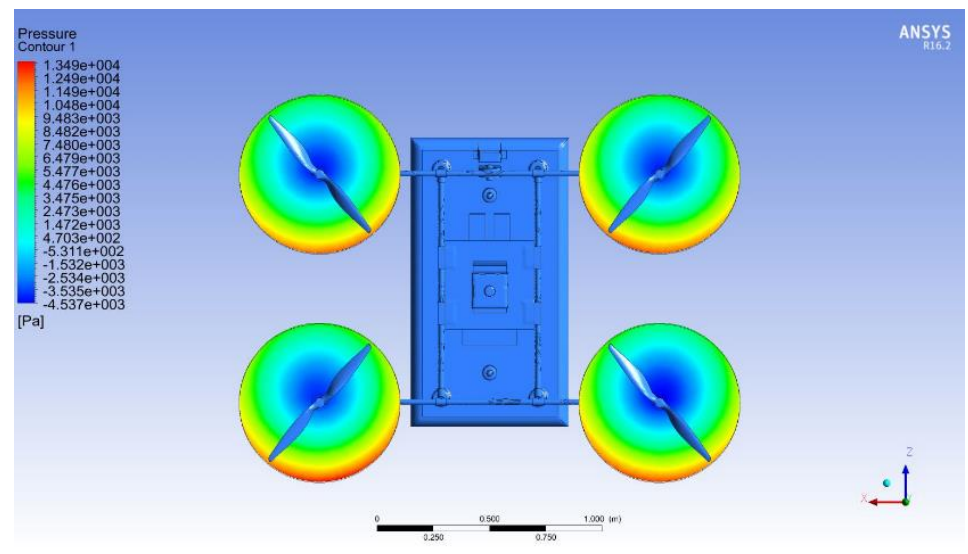

(b) Pressure contour

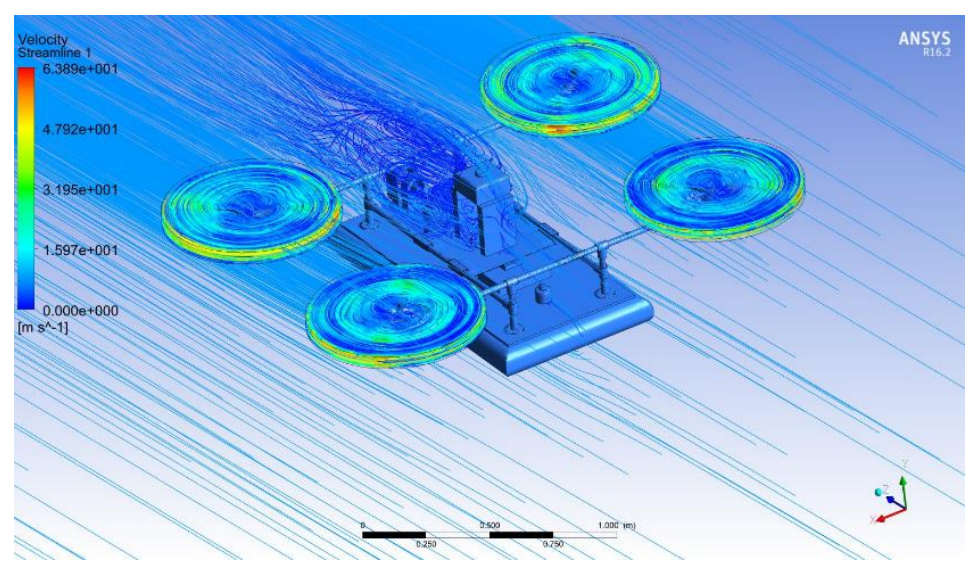

(c) Velocity streamline

Figure 8. Amphibious UAV in forward flight.

\section{CFD Analysis with Canopy}

In order to overcome this issue, a canopy is introduced to the UAV structure as shown in Figure 9. The CFD analysis is performed and simulation results suggested that there is uniform down streamflow for hovering (Figure 10(a)) as well as forward flight (Figure 10(b)) condition. Drag forces are estimated for with and without canopy amphibious models and about $20 \%$ of reduction in drag is obtained at $5^{\circ} \mathrm{AoA}$ (Table 4).

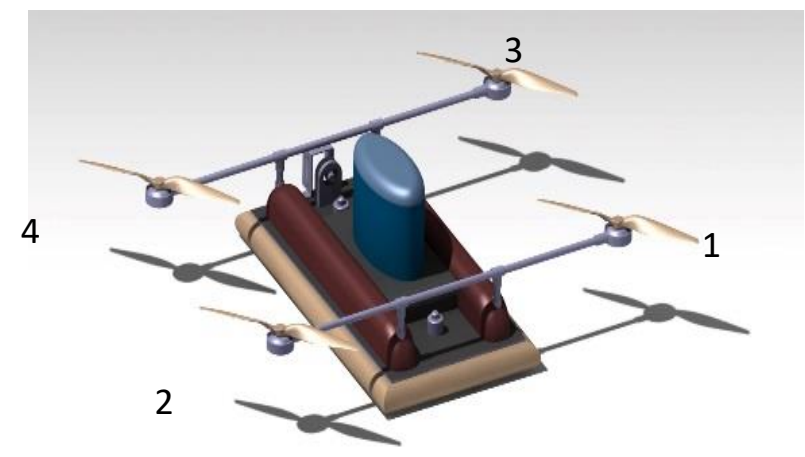

Figure 9. An amphibious model with canopy. 


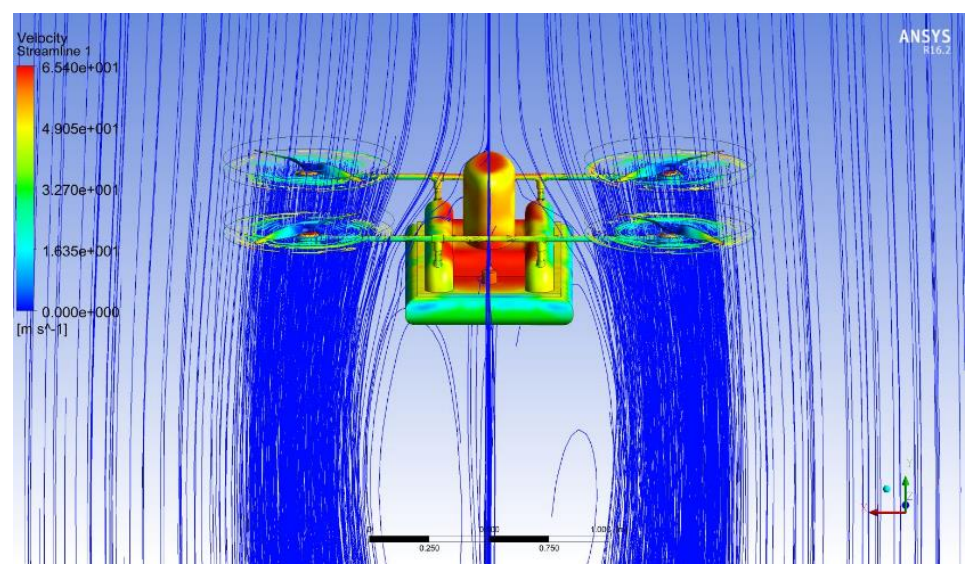

(a) Hovering

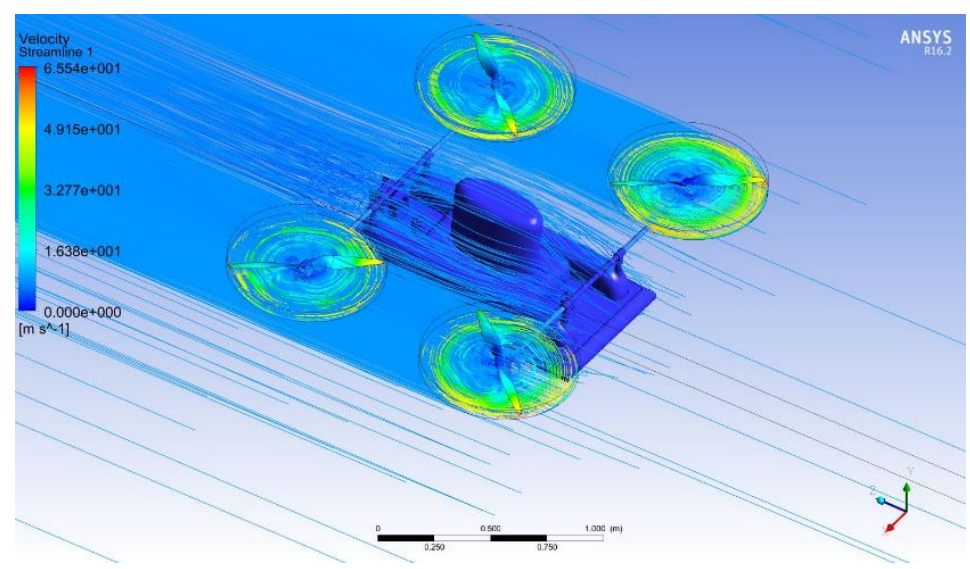

(b) Forward flight

Figure 10. Amphibious UAV with canopy.

Table. 4. Estimation of drag with and without canopy models.

\begin{tabular}{lccc}
\hline AoA $\left(^{\circ}\right)$ & Drag without canopy $(\mathrm{N})$ & Drag with canopy $(\mathrm{N})$ & Drag Reduction $(\%)$ \\
\hline 0 & 7.278 & 6.12 & $15.9 \%$ \\
5 & 7.164 & 5.74 & $19.9 \%$ \\
8 & 7.308 & 6.39 & $12.6 \%$ \\
\hline
\end{tabular}

\section{AERODYNAMIC INTERACTION OF MULTIROTOR WITH CANOPY}

Rotor flow interaction induces vibration in the fuselage and it may lead to instability of the amphibious structure. It is necessary to examine the rotor flow interaction in hovering and forward flight conditions for enhancing the endurance and identify optimal flight parameters. In the multirotor systems, each rotor flow is in close proximity and they may create aerodynamic disturbances to the system. Hence, examining the rotor flow characteristics of multirotor is of major concern. Downwash of rotor impinging on the fuselage creates extra effort and that may reduce the payload capacity. In order to evaluate the aerodynamic interactions among the rotors, the present study focuses on the effect of varying the wind speeds, speed of propellers and ground clearance through performing CFD analysis. 


\section{Effect of Varying Wind Speeds}

At various relative velocities of the amphibious vehicle $(5-12 \mathrm{~m} / \mathrm{sec})$ CFD analysis is performed. From the velocity contours in Figure 12(a) to 12(d), there is a reverse flow region formed at the downstream of the vehicle. Also, due to the curvature profile of the body, higher velocities and acceleration regimes were formed in the upstream of the vehicle. There is stagnation pressure rise in the upstream flow due to wind load acting at the frontal surface. Formation of negative pressure region on the top surface of vehicle is found at various vehicle speeds and also high-pressure boundaries are observed in the downstream of vehicle. The highpressure boundary is not seen at the vehicle speed of $10 \mathrm{~m} / \mathrm{sec}$ in Figure12(c) and the pressure is distributed uniformly. The turbulent kinetic energy is concentrated at the downstream during low vehicle speed of $3 \mathrm{~m} / \mathrm{sec}$ and significant effect is observed up to $10 \mathrm{~m} / \mathrm{sec}$. At $10 \mathrm{~m} / \mathrm{sec}$ the turbulent kinetic energy is distributed and vorticity 3 region is shifted away from the vehicle. Further, increase of vehicle speed causes creation of turbulent kinetic energy region at the downstream of vehicle. Hence, it is recommended to operate the amphibious vehicle between $5 \mathrm{~m} / \mathrm{sec}$ to $10 \mathrm{~m} / \mathrm{sec}$ for better stability.

By varying the wind speed causes an increase in Reynolds number and in such cases co-efficient of thrust is estimated for all four propellers. It is evident from Figure 11 that, at 8.3 $\mathrm{m} / \mathrm{sec}(\operatorname{Re} 83000)$, maximum amount of thrust is generated at each propeller. Further increase in wind velocity resulted in decreasing trend of thrust.

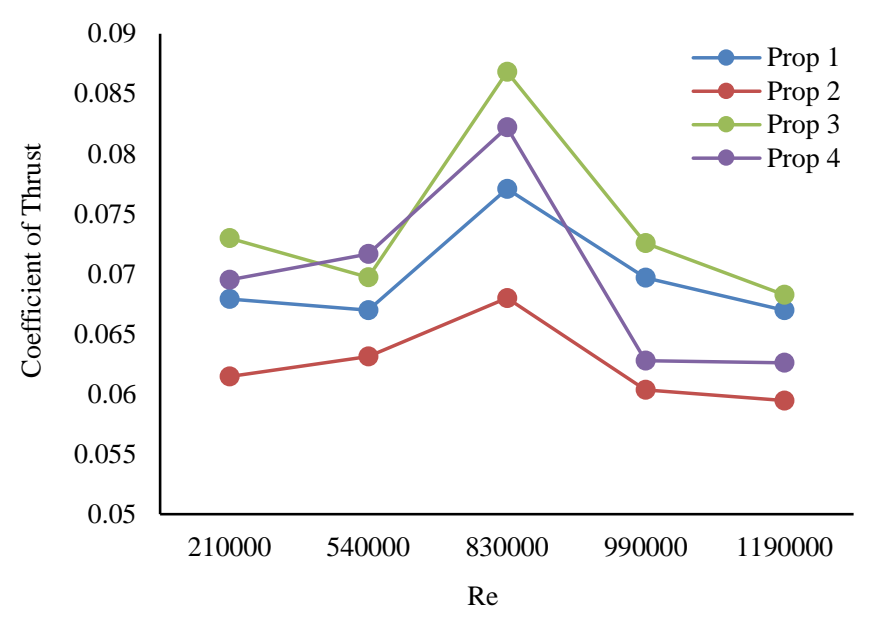

Figure 11. Amphibious UAV in forward flight condition at $5^{\circ}$ AoA. 

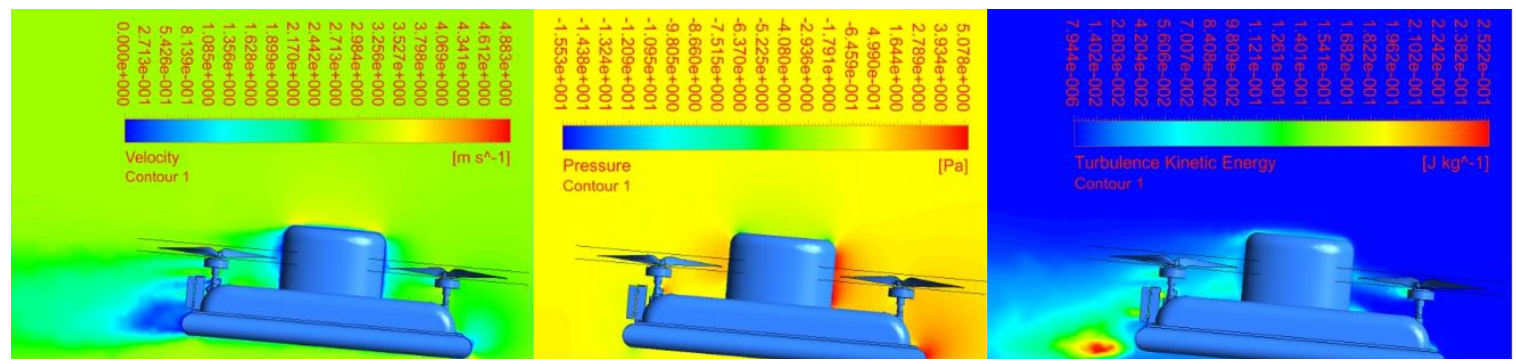

(a) Free stream of velocity at $\mathrm{V}_{\infty} 3 \mathrm{~m} / \mathrm{s}$
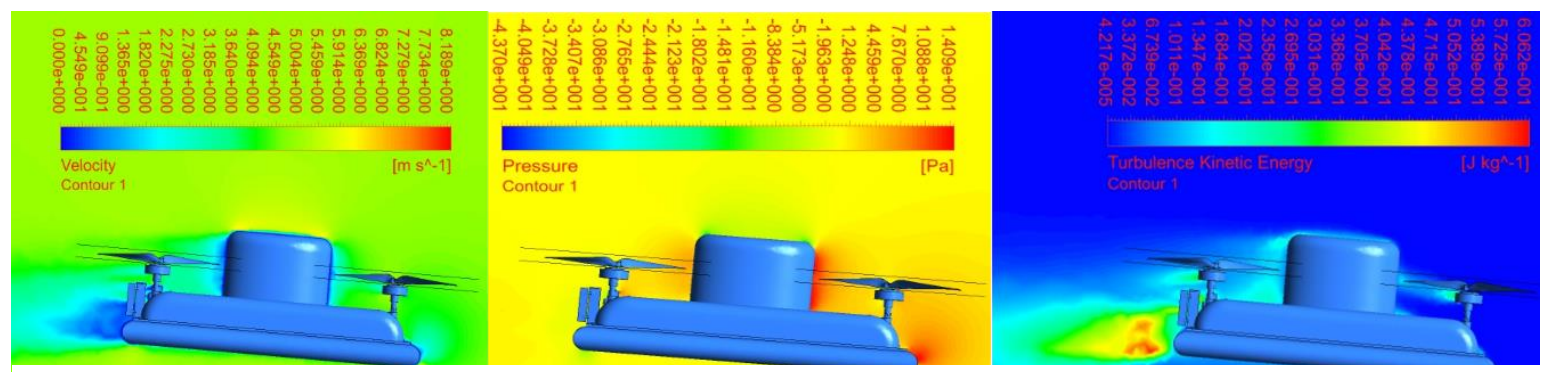

(b) Free stream of velocity at $\mathrm{V}_{\infty} 5 \mathrm{~m} / \mathrm{s}$
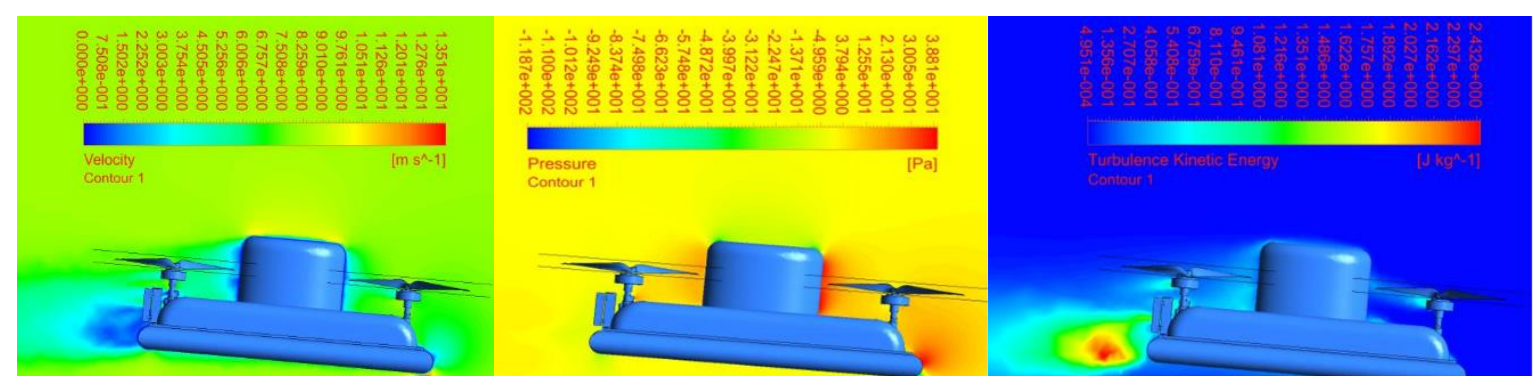

(c) Free stream of velocity at $\mathrm{V}_{\infty} 8.3 \mathrm{~m} / \mathrm{s}$
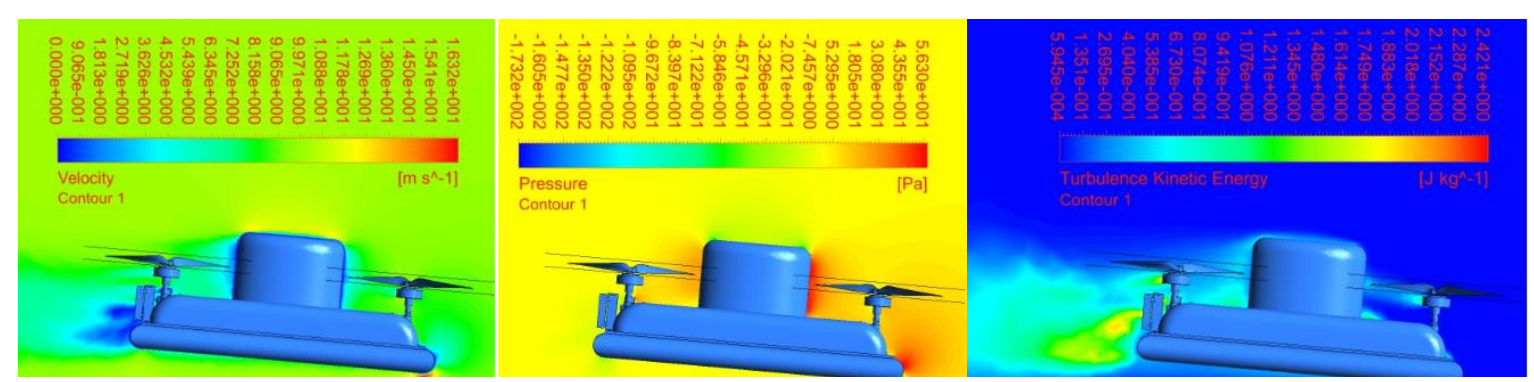

(d) Free stream of velocity of $V_{\infty} 10 \mathrm{~m} / \mathrm{s}$

Figure 12. Effect of relative velocity in flow characteristics.

\section{Effect of Changing the Speed of Propellers}

The effect of speed of propeller with respect to local velocity is calculated as the advance ratio. Increase of propeller speed resulting in decrease in advance ratio and correspondingly thrust increases as depicted in Figure 13(a). At $\mu=0.09$ there is a sudden increase of thrust coefficient 
and further decrease of advance ratio (increase of propeller speed) may not affect the thrust level significantly. However, influence of tip speed of propeller is measured with induced flow ratio which is calculated through performing CFD analysis. As the rotor speed increases cause a decrease in induced inflow and advance ratio as observed in Figure 13(b). As the advance ratio increases, the relative velocity experienced by the retreating blade decreases so that the tip of the blade experiences zero velocity. Hence, the thrust generation of each rotor varied with respect to speed of propeller. The flow interference due to the shape of the structure affects the thrust force generated from each rotor. Since the thrust variations are around less than $5 \%$ in the propellers and we assumed to be negligible.

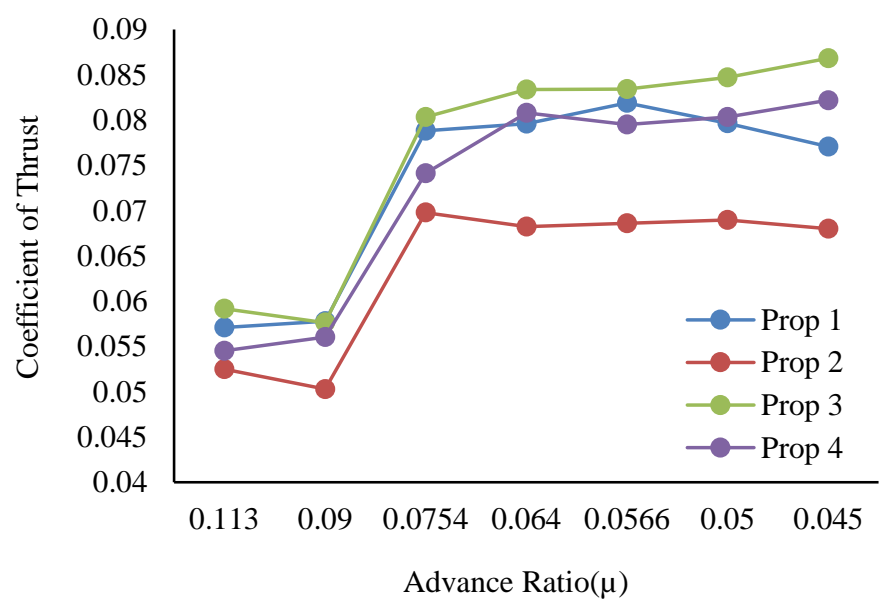

(a) Effect of speed of propeller

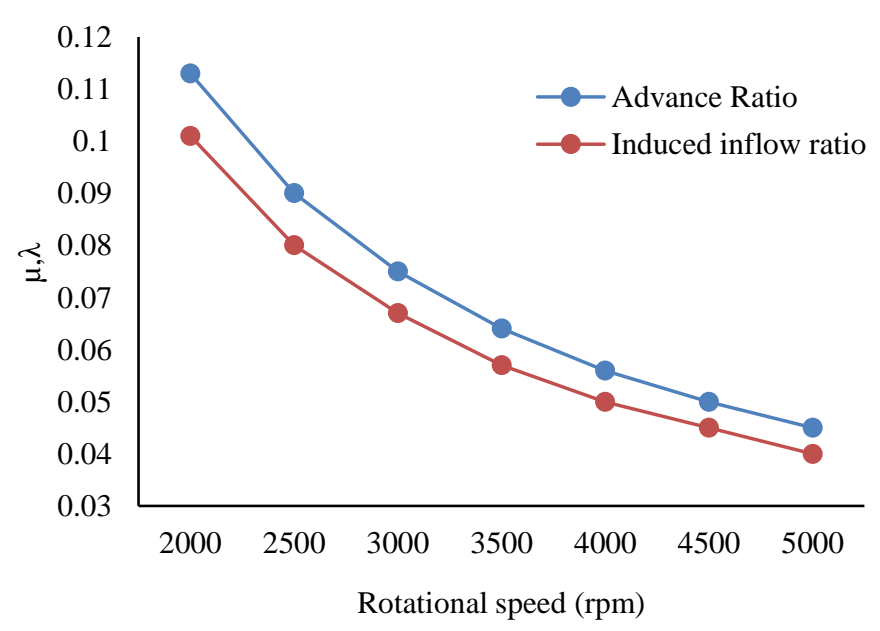

(b) Effect of tip speed of the propeller

Figure 13. Aerodynamic interaction with varying rotor speed.

The induced inflow ratio is measured at the tip path plane for the different rotational speed of propeller and it is observed that all the rotors exhibited similar flow characteristics. The rotor is rotating at counter-clockwise direction and free stream airflow direction is indicated in Figure 14 is considered to be advancing side of the rotor. The induced inflow pattern is moreover similar while increasing the advance ratio. At all advance ratios, the induced inflow is unsymmetrical about the lateral centerline. The predicted induced inflow ratio shows that 
greatest downwash created in the advancing side region of the propeller tip. Due to downwash, there is a large eddies formation in the rotor blade tip which creates the wake interference with the fuselage. However while increasing the advance ratio the downwash is increased accordingly which is identified by level of the induced inflow ratio. As the advanced ratio increases the rollup processes travels towards downstream and correspondingly local induced velocity is increased. It is evident from Figure 14 that the intensity of velocity gradient is high at $\mu=0.045$ (5000 rpm).

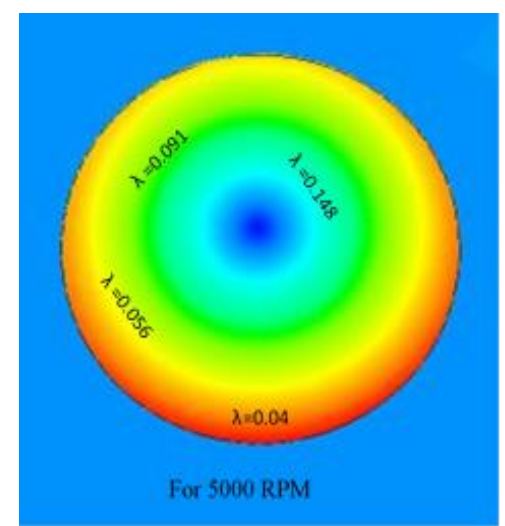

(a) $\mu_{a}=0.045$

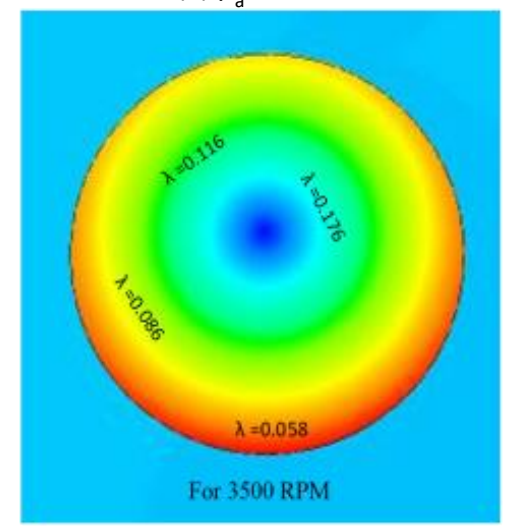

(d) $\mu_{a}=0.064$

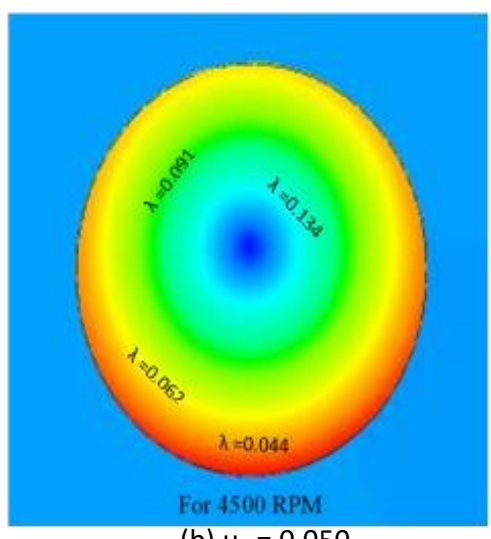

(b) $\mu_{a}=0.050$

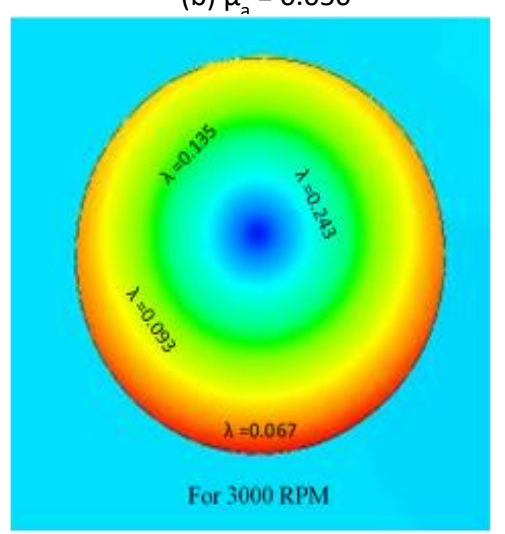

(e) $\mu=0.075$

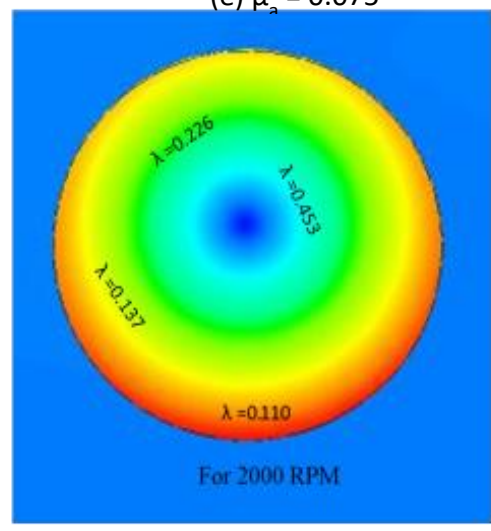

(g) $\mu_{a}=0.113$

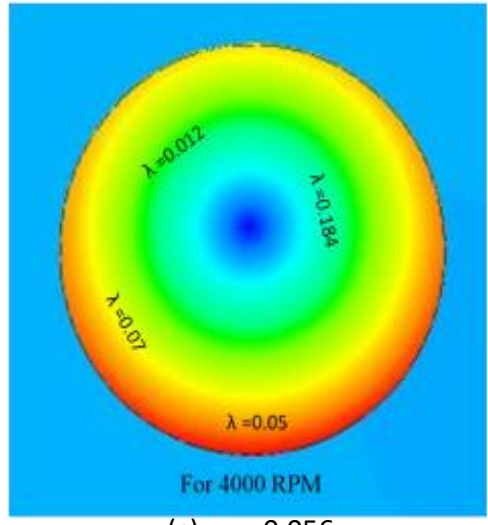

(c) $\mu_{3}=0.056$

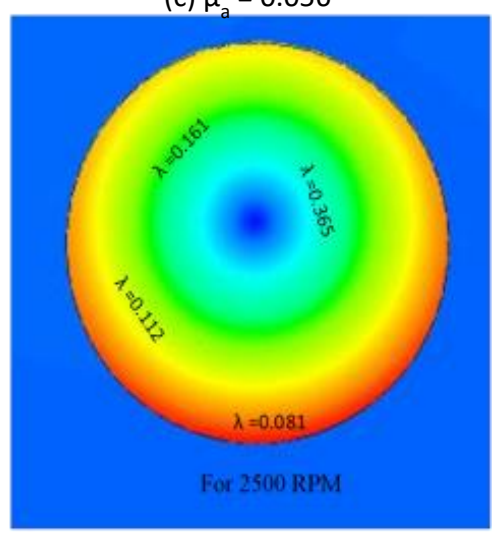

(f) $\mu_{a}=0.091$

Figure 14. Induced inflow ratio for various rotor speeds. 


\section{Pressure Distribution over the Fuselage and Rotor}

In the axisymmetrical plane of amphibious vehicle, pressure coefficient is determined. During forward condition of vehicle the frontal region attained high pressure and due to the obstruction of airflow at the duct fan causes negative pressure gradient. It is further increased due to the water sampler in the region $0.1-0.3$ as depicted in Figure 15. After the water sample region flow gets attached to the fuselage and then separated at the rudder region of the vehicle and hence negative pressure is created as shown in Figure 15. Co-efficient of pressure is determined for a rotor rotating at $5000 \mathrm{rpm}$. It is evaluated for the rotor advancing and retreating side with varied azimuth angle as depicted in Figure 16. In the advancing side positive pressure is experienced and there is negative pressure in the retreating side is observed. Due to the pressure difference, lift of the vehicle is guaranteed. The symmetricity of pressure in the advancing and retreating side of propeller implies the stability of the vehicle.

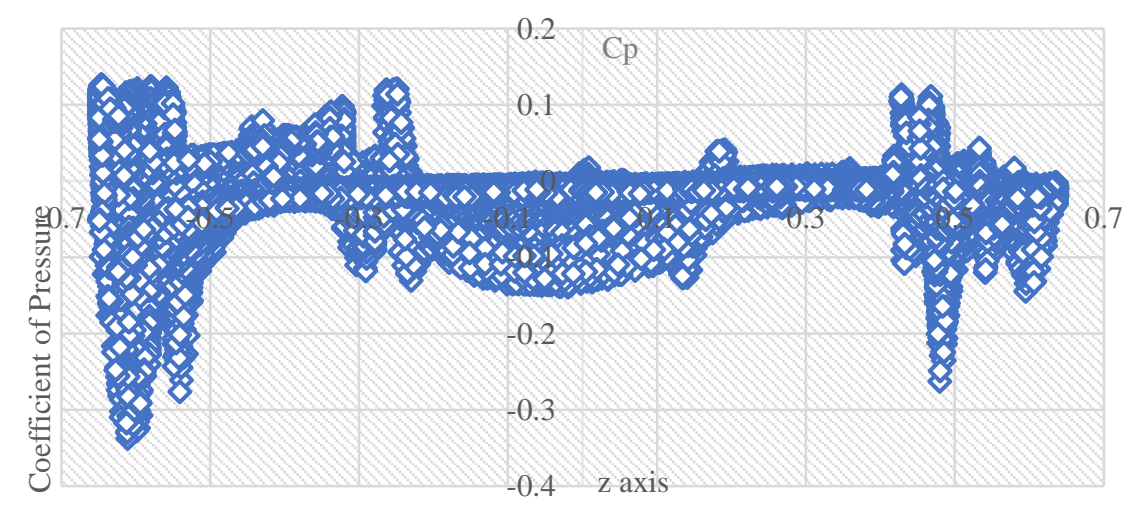

Figure 15. Distribution of pressure along the asymmetrical plane of the amphibious surface.

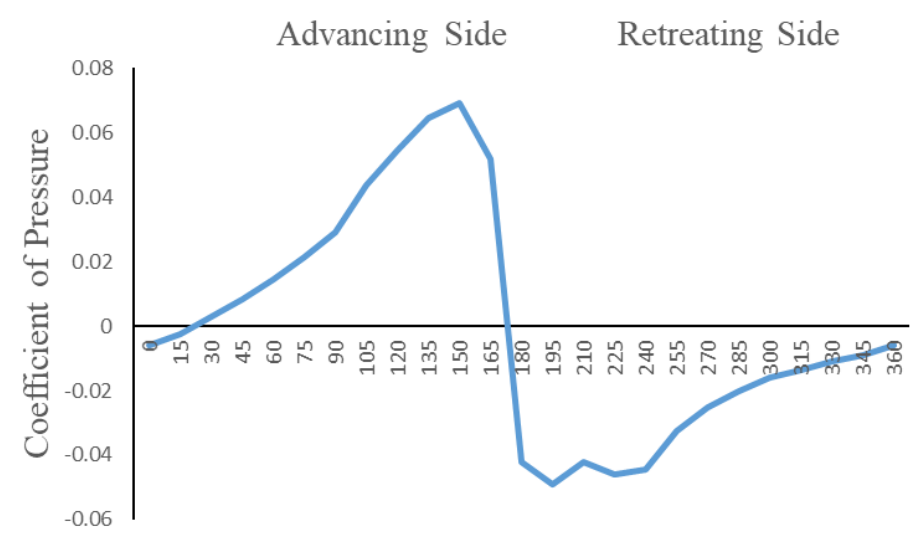

Azimuth angle $(\varphi)$

Figure 16. Distribution of pressure in a rotor.

\section{GROUND EFFECT}

The ground effect has significant impact on hovering capability of the amphibious vehicle and it also plays a vital role in pitch and yaw calibration during take-off ensuring the stability of the vehicle [22-23]. The aerodynamic interaction between propellers and ground surface may have serious concern during slow speed and hovering of the vehicle. The multirotor hovering close 
to the vicinity of ground surface causes rotor wake expands rapidly and the vertical downwash is transformed into radial outwash. Due to this phenomenon, slipstream and induced velocity are altered which subsequently affects the rotor thrust and power.

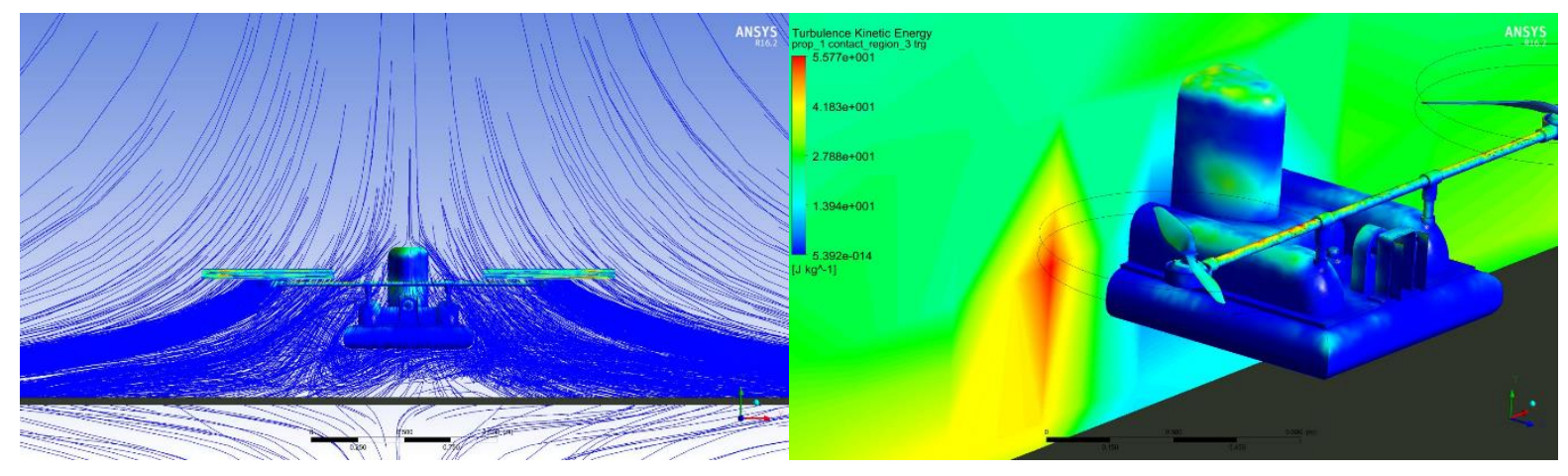

(a) Ground clearance of $1 \mathrm{x}$ propeller diameter

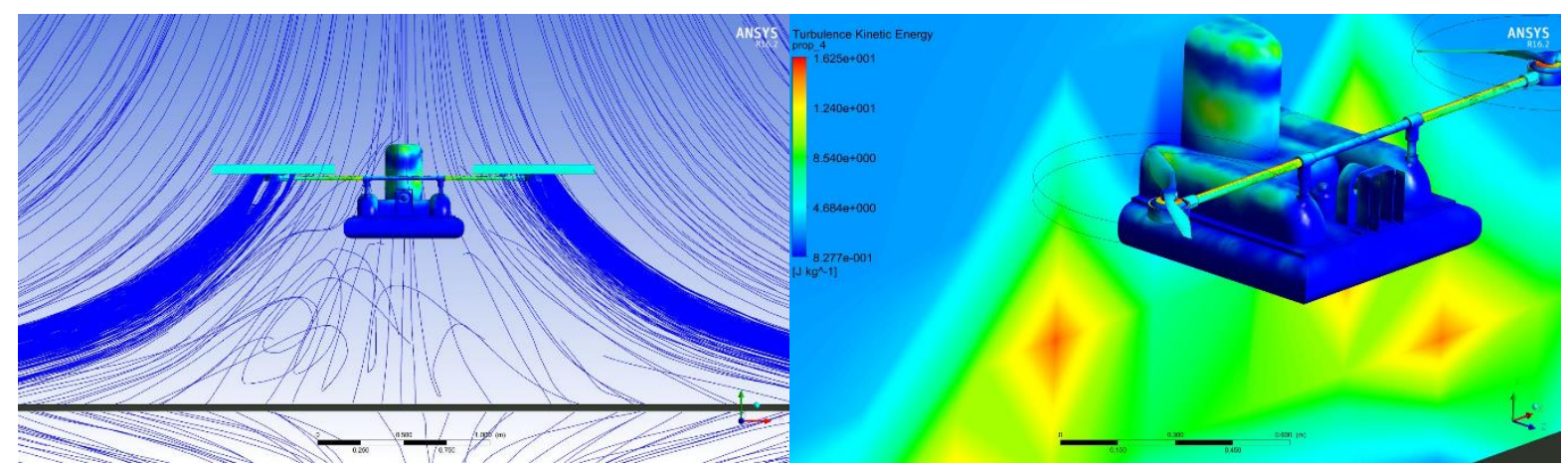

(b) Ground clearance of $2 x$ propeller diameter
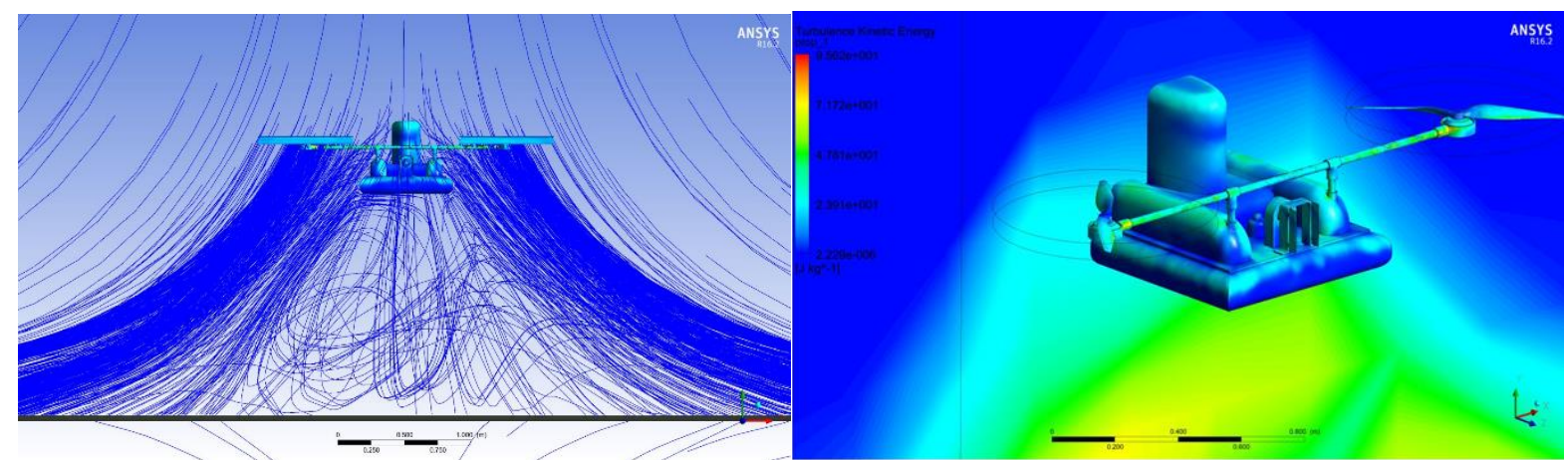

(c) Ground clearance of $3 \times$ propeller diameter

Figure 17. Aerodynamic interaction due to ground effect.

In this study, the interaction effect due to the ground clearance is simulated through varying the distance between the ground and vehicle surface. It is varied with multiplicity of propeller diameter up to three times (Figure 17). It is observed that at one-time propeller diameter there is a huge vortex region is formulated and it is reduced when the distance is increased. At three times propeller diameter, the turbulent kinetic energy is decreased and hence the ground effect is predominant in the case of two times propeller diameter and below. There is a recirculation of flow which causes increase of thrust due to the effect of ground is observed 
in each propeller as seen in Figure 17. The generation of thrust due to the ground effect is depicted in Figure 18 which describes that increase in the distance between ground and vehicle achieved decrease in thrust and is almost uniform in all propellers.

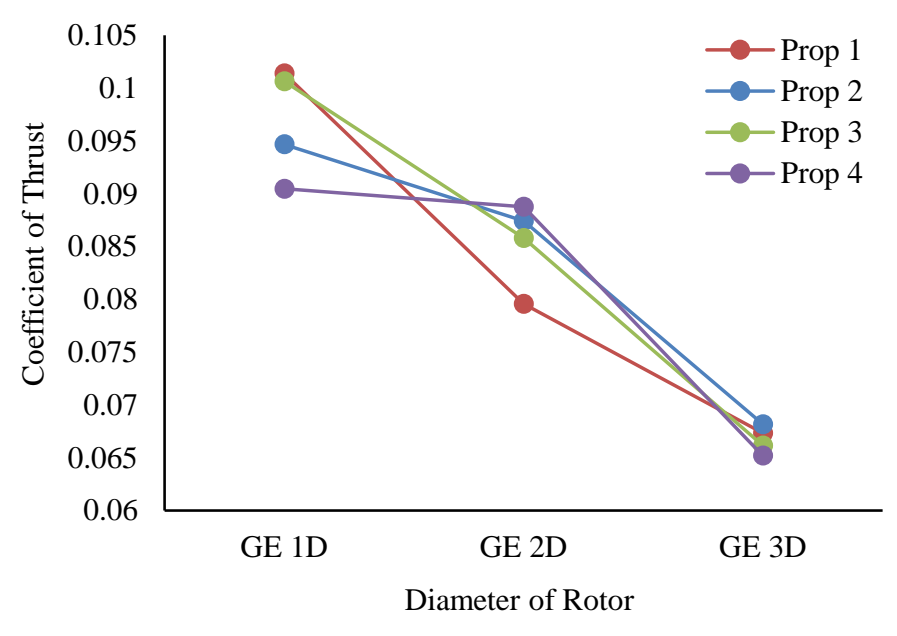

Figure 18. Thrust characteristics due to ground effect.

\section{CONCLUSION}

In this study, aerodynamic interactions of novel amphibious vehicle in forward flight and hovering conditions are examined using CFD analysis. Among the various turbulent models, SST k- $\omega$ model obtained minimal error of $1 \%$ in predicting the drag in comparison to other models. This model is able to predict the turbulence level, wake, bound vortex region, and thrust and pressure coefficients using moving reference frame method in steady flow conditions. At $5^{\circ} \mathrm{AoA}$ and $8.3 \mathrm{~m} / \mathrm{sec}$ relative velocity of the vehicle, minimal drag force is obtained by amphibious vehicle. The CFD studies through incorporating canopy in the amphibious structure resulted in $20 \%$ of reduction in drag. Interaction effect on varying the propeller speed from $2000 \mathrm{rpm}$ to $5000 \mathrm{rpm}$ is analysed and it is concluded that at $5000 \mathrm{rpm}$ tremendous downwash is produced at the advancing side of the rotor which is evident from the induced inflow ratio of 0.04 and at $3500 \mathrm{rpm}$ uniform flow characteristics are observed. At Re of 83000, maximum amount of thrust is produced and hence amphibious vehicle can be operated at a relative velocity of $8.3 \mathrm{~m} / \mathrm{sec}$ to achieve better aerodynamic performance. Ground effect phenomenon through varying the ground clearance with respect to change in multiplicity of diameter of the propeller resulted that three times of propeller diameter obtained uniform distribution of turbulent intensity leads to better equilibrium of the vehicle. Also, symmetrical pressure distribution along the fuselage and rotor guaranteed the equilibrium of the vehicle while operating at $5^{\circ}$ AoA and $8.3 \mathrm{~m} / \mathrm{sec}$.

\section{REFERENCES}

[1] Antoniadis AF, Drikakis D, Zhong B, Barakos G, et al. Assessment of CFD methods against experimental flow measurements for helicopter flows. Aerospace Science and Technology 2012; 19(1):86-100. 
[2] Aich S, Ahuja C, Gupta T, Arulmozhivarman P. Analysis of ground effect on multirotors. In: International Conference on Electronics, Communication and Computational Engineering (ICECCE), pp. 236-241; 2014.

[3] Biava M, Khier W, Vigevano L. CFD prediction of air flow past a full helicopter configuration. Aerospace Science and Technology 2012; 19(1):3-18.

[4] Boyd, JrD, Barnwell R, Gorton SA computational model for rotor-fuselage interactional aerodynamics. In 38th Aerospace Sciences Meeting and Exhibit, pp. 256; 2000.

[5] Cao YH, Yu ZQ, Yuan SU, Kai KA. Combined free wake/CFD methodology for predicting transonic rotor flow in hover. Chinese Journal of Aeronautics 2002; 15(2):6571.

[6] Celic A, Hirschel EH. Comparison of eddy-viscosity turbulence models in flows with adverse pressure gradient. Aiaa Journal 2006; 44(10):2156-69.

[7] Conlisk AT. Modern helicopter rotor aerodynamics. Progress in aerospace sciences 2001; 37(5):419-76.

[8] Dindar M, Shephard MS, Flaherty JE, Jansen K. Adaptive CFD analysis for rotorcraft aerodynamics. Computer methods in applied mechanics and engineering 2000; 189(4):1055-76.

[9] Domenge PX, Ilie M. Numerical study of helicopter blade-vortex mechanism of interaction using the potential flow theory. Applied Mathematical Modelling 2012; 36(7):2841-57.

[10] Felismina R, Silva M, Mateus A, Malça C. Study on the aerodynamic behavior of a UAV with an applied seeder for agricultural practices. In: AIP Conference Proceedings, pp. 020049; 2017.

[11] Filippone A, Michelsen JA. Aerodynamic drag prediction of helicopter fuselage. Journal of Aircraft 2001; 38(2):326-33.

[12] Hassanalian M, Abdelkefi A. Classifications, applications, and design challenges of drones: A review. Progress in Aerospace Sciences 2017; 91:99-131.

[13] Lesieur M. Turbulence in fluids: stochastic and numerical modelling. Boston: Martinus Nijhoff; 1987.

[14] Lopez OD, Escobar JA, Pérez AM. Computational Study of the Wake of a Quadcopter Propeller in Hover. In: 23rd AIAA Computational Fluid Dynamics Conference, pp. 3961; 2017.

[15] Qijun ZH, Guoqing ZH, Bo WA, Qing WA, Yongjie SH, Guohua XU. Robust NavierStokes method for predicting unsteady flowfield and aerodynamic characteristics of helicopter rotor. Chinese Journal of Aeronautics 2018; 31(2):214-24.

[16] Sanchez-Cuevas P, Heredia G, Ollero A. Characterization of the aerodynamic ground effect and its influence in multirotor control. International Journal of Aerospace Engineering 2017; 2017: 1823056.

[17] Shi Y, Xu Y, Xu G, Wei PA coupling VWM/CFD/CSD method for rotor airload prediction. Chinese Journal of Aeronautics 2017; 30(1):204-15.

[18] Steijl R, Barakos GN. Computational study of helicopter rotor-fuselage aerodynamic interactions. AIAA Journal 2009; 47(9):2143-57.

[19] Tan J, Wang H. Panel/full-span free-wake coupled method for unsteady aerodynamics of helicopter rotor blade. Chinese Journal of Aeronautics 2013; 26(3):535-43.

[20] Thibault S, Holman D, Garcia S, Trapani G. CFD Simulation of a quad-rotor UAV with rotors in motion explicitly modeled using an LBM approach with adaptive refinement. In: 55th AIAA Aerospace Sciences Meeting, pp. 0583; 2017. 
[21] Versteeg HK, Malalasekera W. An introduction to computational fluid dynamics: The finite volume method. PEARSON Prentice Hall; 2007.

[22] Ye L, Zhang Y, Yang S, Zhu X, Dong J. Numerical simulation of aerodynamic interaction for a tilt rotor aircraft in helicopter mode. Chinese Journal of aeronautics 2016; 29(4):843-54.

[23] Yoon S, Lee HC, Pulliam TH. Computational analysis of multi-rotor flows. In: 54th AIAA Aerospace Sciences Meeting, pp. 0812; 2016. 